Nloman 2019, 37(1), 45-53

Revista de Psicologia, Ciències de l'Eduació i de l'Esport

ISSN: $1138-3194$

CFacultat de Psicologia, Ciències de l'Educació i de l'Esport Blanquerna

Universitat Ramon Llull

\title{
Aprendizaje entre iguales y desarrollo profesional de los estudiantes en prácticas del Grado de Educación Primaria a través de los foros online
}

\author{
Miryam Martínez-Izaguirre, Lucía Barrenetxea \& Ariane Díaz-Iso \\ Universidad de Deusto \\ Recibido: 17 sept 2018 \\ Aceptado:30 jan 2019
}

Aprendizaje entre iguales y desarrollo profesional de los estudiantes en prácticas del Grado de Educación Primaria a través de los foros online

\begin{abstract}
Resumen. Este trabajo presenta el impacto del empleo de los foros online sobre la mejora de los aprendizajes en las prácticas profesionales de los futuros docentes de educación primaria. Para ello, se analiza el uso de este recurso por parte de los estudiantes y su percepción sobre la utilidad e idoneidad de esta herramienta para lograr el máximo aprovechamiento de la experiencia de prácticas. Los resultados derivados del análisis cuantitativo de las interacciones registradas en los foros, así como del análisis cualitativo sobre las respuestas otorgadas a un cuestionario de carácter abierto orientado a recoger la valoración del uso del foro como herramienta de aprendizaje en el prácticum, revelan un elevado grado de satisfacción con las posibilidades que este recurso ofrece. Entre las más destacables se sitúan: las oportunidades para la interacción y el aprendizaje entre iguales en un contexto de aprendizaje que por la distancia física limita procesos colaborativos; la calidad del apoyo y orientación recibidos en momentos de práctica que requiere una respuesta inmediata; las posibilidades para mejorar la regulación emocional ante situaciones de aprendizaje en entornos reales, así como profundizar en la reflexión sobre la práctica y las competencias docentes implementadas, indispensable para el desarrollo profesional.
\end{abstract}

Palabras clave: foro online; aprendizaje entre iguales; prácticum; regulación emocional; desarrollo profesional

Peer learning and professional development of students in the Primary Education Degree through the online forums

Summary. This paper presents the impact of the use of online forums on the improvement of learning in the professional training of prospective teachers of Primary Education. For this purpose, we analyse the use of this resource from the students' perspective and examine their perception of the tool's usefulness in helping them to get as much as possible out of their teaching practice. Results were obtained not only from the quantitative analysis of the interactions recorded in the forums, but also from the qualitative analysis of the answers students provided to an open-ended questionnaire that assessed the use of the forum as a learning tool in the Practicum. Our findings reveal that many students were engaged with the forum and that their assessment of the range of possibilities offered by this resource was positive. The possibilities that the students were most likely to highlight were: the opportunity for interaction and peer learning in a context where collaborative processes are limited by physical distance; the support and guidance received at times when an immediate response is required; the possibility to regulate emotions and deepen their reflections on the professional practice and teaching skills that are indispensable for professional development.

Keywords: online forum; peer learning; professional training; emotional regulation; professional development

Correspondencia

Ariane Díaz-Iso

Universidad de Deusto

ariane.diaz.iso@deusto.es 


\section{Introducción}

La preparación de docentes competentes capaces de responder a los retos educativos derivados de los desafíos sociales actuales es la meta prioritaria en la formación inicial de los futuros maestros. Siendo esta una tarea compleja para abordar en la titulación del Grado de Educación Primaria, los periodos de prácticas resultan un marco incomparable para su logro. De hecho, el prácticum es el primer escenario que pone a prueba y exige: aplicar las competencias desarrolladas a lo largo del título, gestionar emocionalmente las realidades que diariamente se presentan desafiando a las propias habilidades docentes y aprovechar los escenarios y situaciones cambiantes para perfeccionar sus competencias.

No obstante, a pesar de la bondad de la experiencia práctica, los amplios espacios de tiempo de permanencia en los centros escolares y el abordaje individual de la tarea, dificultan la implementación de cuatro dimensiones esenciales para el éxito en el aprendizaje:

- La interacción con los iguales, para enriquecerse y aprender de manera colaborativa. El escaso contacto directo entre compañeros limita sacar el máximo partido a la experiencia.

- Disponer de un apoyo, acompañamiento y seguimiento, constantes y estables en el tiempo, por parte del tutor de prácticas. Aunque el alumnado acude a seminarios presenciales en la universidad, estos son insuficientes para ofrecer un apoyo sólido.

- Disponer de espacios para el apoyo en la gestión y regulación emocional, necesarias para el abordaje de situaciones nuevas y complejas de la actividad escolar diaria, que retan a las propias competencias docentes desarrolladas hasta el momento.

- Encontrar espacios para reflexionar eficazmente sobre la acción, para mejorar la praxis docente (Schön, 1983) y la consolidación de las competencias básicas de la profesión (Martínez-Izaguirre, Yániz \& Villardón, 2018).

Ante estas limitaciones, se consolida la necesidad de aprovechar el apoyo que ofrece la tecnología, utilizando nuevos escenarios educativos de carácter virtual que superen el aula física (Gisbert, 2013), seleccionando y usando recursos proactivos. Entre los recursos existentes, el foro online se muestra como una herramienta funcional para las prácticas docentes, pues favorece el debate, el feedback, el intercambio de experiencias, las problemáticas, las inquietudes, el apoyo entre iguales y la colaboración en el aprendizaje (Romero López, Luna \& Ventura, 2013). Asimismo, ayuda a construir conocimiento a través de la interacción online (Loncar, Barrett \& Liu; 2014; Tirado, Hernando \& Aguaded, 2015), y a desarrollar la competencia en comunicación escrita y de reflexión (Ornelas, 2007).

Por todo ello, se observa la necesidad de integrar esta herramienta en el apoyo y seguimiento de los es- tudiantes de prácticum del Grado de Educación Primaria. Su inclusión resulta particularmente útil porque permite cubrir las necesidades formativas en tiempo real, sin esperar a momentos de encuentro presencial que quizás ya no resuelvan dificultades que la práctica arroja diariamente.

Atendiendo a las necesidades formativas de los estudiantes de prácticum del Grado de Educación Primaria, y a las oportunidades que podía ofrecer el foro en el seguimiento y apoyo de sus experiencias y aprendizajes, durante el curso 2017-2018 se ha aplicado este recurso en el seguimiento de los dos periodos de prácticas asociados a esta titulación, Prácticum I y Prácticum II, en la Universidad de Deusto. Su incorporación pretendió lograr cuestiones que la innovación educativa ha mostrado esenciales para la mejora del aprendizaje en los últimos años:

- Mejorar los procesos de interacción entre profesor y estudiante (Carbonell, 2005; Escudero, 2006).

- Favorecer una mayor interacción entre iguales (Topping, 1996; Durán \& Vidal, 2004; Kokotsaki \& Hallam, 2007; McFarlane, Spes-Skrbis \& Taib, 2017), que permita compartir experiencias, sentimientos y promueva un aprendizaje colaborativo.

- Incorporar los avances tecnológicos para responder de manera eficaz a necesidades educativas sentidas (Sanabria \& Hernández, 2011); en este caso, las referidas a las experiencias de prácticas.

Para ello, inicialmente se construyó un espacio virtual en el seno de la plataforma Moodle, que albergaba tres foros diferenciados y con tres fines claros:

- Primer foro: aprender a gestionar y regular las emociones derivadas de las actuaciones en contextos profesionales reales.

- Segundo foro: construir conocimiento profesional a partir del aprendizaje colaborativo sobre prácticas educativas de éxito.

- Tercer foro: reflexionar sobre las funciones y competencias que hay que emplear en la profesión docente para favorecer el desarrollo profesional.

La introducción de este recurso ha contemplado que el mero hecho de utilizar la herramienta no es en sí mismo garantía de aprendizaje. Y por ello, se ha velado en todo momento por el mantenimiento de unas condiciones básicas que favorecieran la participación de los estudiantes en él, como una actuación constante del docente y ante momentos significativos para los estudiantes, atendiendo a las recomendaciones de Parks-Stamm, Zafonte y Palenque (2017). Asimismo, contemplando las aportaciones de Loncar et al. (2014), y de Tan (2017), se ha realizado un seguimiento activo de las discusiones y publicaciones de los estudiantes en el foro con el fin de promover una construcción de conocimiento eficaz.

Mediante el estudio que a continuación se presenta, se analiza la percepción sobre la utilidad del foro en los estudiantes que lo han empleado, así como su grado de satisfacción sobre los beneficios que su uso les ha reportado en el logro de los objetivos forjados en el prácticum. 


\section{Método}

La investigación llevada a cabo pretende analizar el grado de implicación de los estudiantes del Grado de Educación Primaria en el uso de los foros online durante las prácticas docentes como futuros maestros, así como su percepción sobre la utilidad y los beneficios que le otorgan. A continuación, se detallan los elementos del diseño metodológico de la investigación realizada para lograr el objetivo planteado.

\section{Participantes}

La muestra está conformada por 36 estudiantes de Grado de Educación Primaria que realizan prácticas durante el curso 2017-2018 en la Universidad de Deusto a quienes, desde la tutoría universitaria, se les da la oportunidad de emplear el recurso del foro como apoyo en su aprendizaje. De ellos, 19 (52.8\%) cursan el Prácticum I, asignatura que favorece un primer contacto con la realidad práctica de la profesión docente en la educación primaria. Los 17 restantes (47.2\%) cursan el Prácticum II - Mención en Pedagogía Terapéutica. Los estudiantes que conforman este grupo son graduados en Educación Primaria y están realizando una segunda mención. Son, por tanto, estudiantes con un importante recorrido formativo y con una experiencia práctica previa en la profesión.

\section{Instrumento}

En este estudio se han empleado dos instrumentos diferentes para la recogida de información. Por un lado, el espacio virtual construido para el foro dentro de la plataforma Moodle, ha permitido recoger el grado de participación de los estudiantes en el foro; así como el tipo de participación realizada en él.

Por otro lado, y con el fin de identificar la percepción que los estudiantes tienen del foro como herramienta de aprendizaje, se ha empleado un cuestionario, sometido previamente a juicio de expertos para garantizar su validez, tal y como proponen Escobar-Pérez y Cuervo Martínez (2008). En él tomaron parte 6 jueces, con experiencia en el seguimiento de procesos de prácticas, así como en el uso de recursos tecnológicos orientados a mejorar los procesos de aprendizaje.

El cuestionario resultante ha contemplado 10 preguntas referidas a la utilidad del foro y su potencialidad para promover la gestión emocional, la colaboración y el aprendizaje entre iguales, así como la reflexión docente. Además, ha recogido la satisfacción con el uso del recurso y, en el caso del alumnado que ya había realizado prácticas previamente, el posible valor añadido en relación con el aprovechamiento de la experiencia de prácticas.

\section{Procedimiento}

A lo largo del proceso de prácticas se realizó un registro semanal del volumen de participación de los estudian- tes en el espacio virtual creado, por medio del cómputo global del número de comentarios realizados, así como registrando su preferencia por participar en cada uno de los tres foros habilitados. Una vez finalizado el proceso de prácticas, los estudiantes cumplimentaron de forma anónima y voluntaria el cuestionario diseñado. Este proceso se realizó de modo online, facilitando el link de acceso a través de un email. De este modo, se otorgaba al alumnado interesado, flexibilidad para responderlo en el momento que les resultara más oportuno.

\section{Análisis de datos}

El análisis de los datos se ha realizado tanto de manera cuantitativa como cualitativa. Por un lado, se han realizado análisis descriptivos de tendencia central y dispersión, así como de frecuencias y porcentajes, en relación con el nivel y tipo de participación de los estudiantes en los foros, información recogida de la plataforma Moodle.

Por otro lado, se ha realizado un análisis cualitativo de las respuestas recogidas a través del cuestionario, mediante la herramienta Atlas.ti. Las respuestas anónimas dadas por los estudiantes fueron añadidas como dos elementos primarios de la Unidad Hermenéutica, diferenciando así las aportaciones de los estudiantes del Prácticum I y del Prácticum II.

Mediante la herramienta de nube de palabras se realizó un rastreo inicial y se identificaron las palabras que se repetían con mayor frecuencia. Estas palabras fueron agrupadas en bloques, con base en la idea que transmitían, posibilitando una aproximación inicial a la percepción de los estudiantes sobre el uso del foro y el conocimiento sobre los aspectos más destacados en él.

Con el fin de profundizar en el análisis, se hizo una categorización en Atlas.ti con base en las dimensiones del cuestionario. Este procedimiento permitió obtener los resultados que se detallan a continuación.

\section{Resultados}

A continuación, se presentan los principales resultados obtenidos en este estudio. Para ello, primeramente, se recogen los resultados de participación de los estudiantes, diferenciándolos en función de la modalidad de prácticum cursada, así como del tipo de participación realizada. Seguidamente, se presentan los resultados obtenidos de las respuestas otorgadas al cuestionario realizado.

\section{Implicación y participación en los foros de discusión creados para el prácticum}

Se observa una alta participación por parte de los estudiantes, registrándose en total 245 publicaciones en el foro, de las cuales el $40.4 \%$ se ha llevado a cabo en el Prácticum I y el 59.6\% en el Prácticum II. Estos datos reflejan una mayor implicación en el uso del 


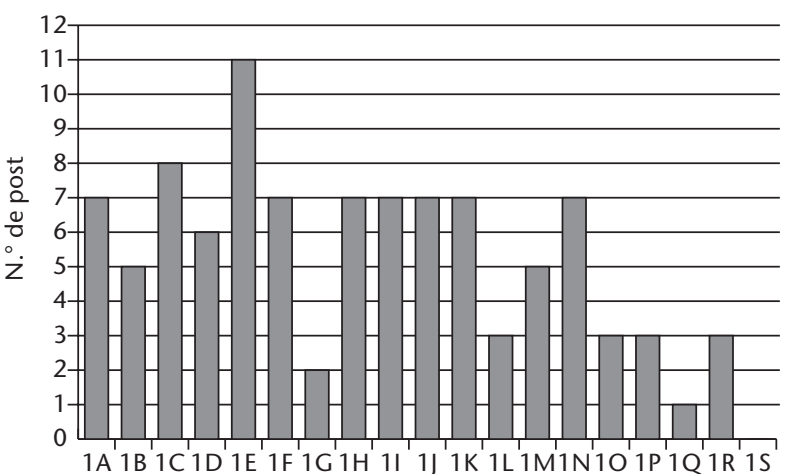

Figura 1. Participación media del alumnado del Prácticum I.

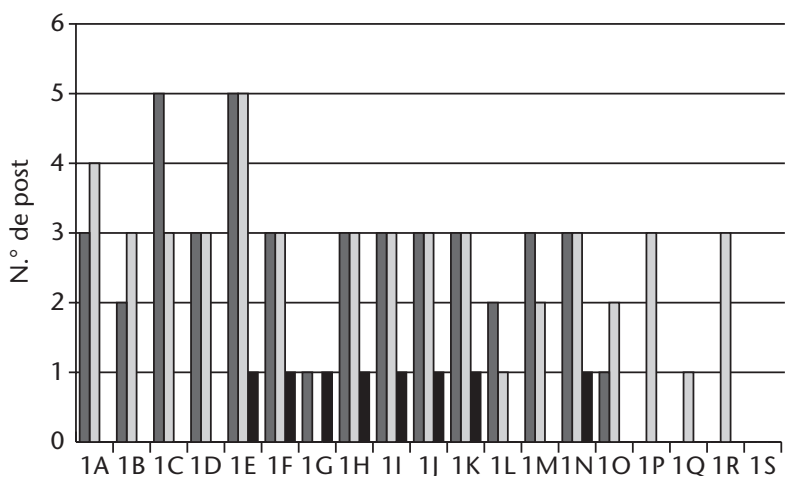

Figura 2. Participación del alumnado del Prácticum I en cada uno de los foros.

foro por parte del alumnado que cursa el Prácticum II.

En el Prácticum I, los estudiantes participan una media de 5.2 intervenciones a lo largo del tiempo de prácticas. Sin embargo, no es homogéneo el grado de participación entre ellos ( $\mathrm{DT}=2.78)$. El dato más elevado se sitúa en 11 intervenciones y el 52\% de este alumnado supera la media de participación. A continuación, se presentan gráficamente los datos de participación de los estudiantes en el Prácticum I (ver figura 1).

El alumnado del Prácticum I, por lo general, ha hecho sus publicaciones en el primer (43.43\%) y segundo foro (48.48\%), que se han convertido estos dos en los foros más frecuentados. El tercer foro, con un $8.08 \%$ de participación, por el contrario, se convierte en el menos transitado. La distribución de las intervenciones de los estudiantes entre los diferentes foros activados puede verse en la figura 2 .

En el Prácticum II, a pesar de que el grupo es más reducido, la media de intervenciones del alumnado ha sido mayor que en el Prácticum I, con 8.5 publicaciones por persona. El $47.05 \%$ ha colaborado más de 8 veces. Además, el $23.5 \%$ ha llevado a cabo entre 12 y 18 participaciones (ver figura 3 ).

Además, se observa un mayor equilibrio en la participación entre los distintos foros, obteniendo un $37.67 \%$ el primero, un $41.78 \%$ el segundo y un $20.54 \%$ el tercero. Se ha de destacar, además, un incremento significativo en la participación del tercer foro, con respecto a los estudiantes del Prácticum I (ver figura 4).

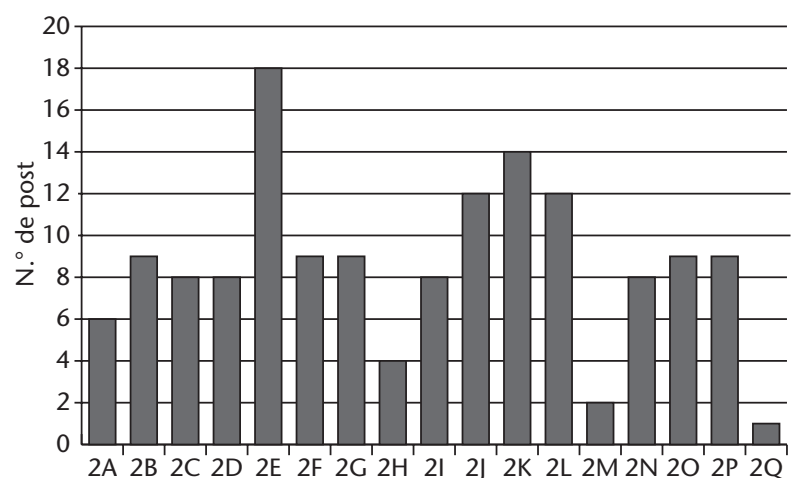

Figura 3. Participación media del alumnado del Prácticum II.

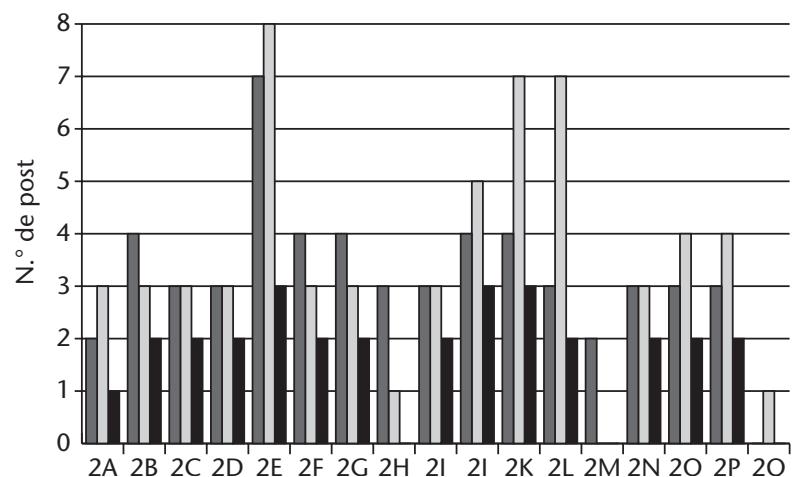

Figura 4. Participación del alumnado del Prácticum II en cada uno de los foros.

En otro orden, un análisis inicial de la nube de palabras resultante, muestra consenso entre los estudiantes a la hora de valorar la utilidad del foro en los procesos de prácticas, y una percepción compartida sobre el recurso entre los dos grupos que conformaban la muestra. En la tabla 1, se registran de manera agrupada las palabras más repetidas, ordenadas en función de la frecuencia de aparición.

Este primer análisis permitió constatar que las dimensiones para analizar a través del cuestionario quedaban ampliamente representadas y valoradas. Seguidamente, los resultados obtenidos se organizaron en las siguientes categorías.

\section{Utilidad del foro en el prácticum}

La valoración que el alumnado hace sobre la utilidad del foro en el prácticum es muy positiva. Existe un alto consenso entre el alumnado (93.10\%) a la hora de afirmar que el foro ha resultado ser una herramienta muy útil. Las razones que los participantes exponen son variadas y coinciden con las categorías analizadas mediante el cuestionario.

Por una parte, el alumnado define el foro como una herramienta para la reflexión: "Poder llevar a cabo nuevas reflexiones que no nos habíamos planteado anteriormente hacer" (A4). Asimismo, afirman que el uso del foro les brinda la oportunidad de ver, compartir y conocer otras experiencias: "Creo que es una buena opción para ver distintas realidades, que en tu centro de trabajo igual no hay" (B5). El apoyo mutuo 
Tabla 1. Aproximación a las ideas más frecuentes sobre el uso del foro en el prácticum a partir de la nube de palabras

\begin{tabular}{|c|c|}
\hline Prácticum I & Prácticum II \\
\hline $\begin{array}{l}\text { Utilidad para la mejora }(f=304) \\
\text { Mejorar }(f=59) \text {, importancia }(f=55) \text {, utilidad }(f=53) \text {, posibilidad }(f=35) \text {, } \\
\text { pertinentes }(f=28) \text {, enriquecer }(f=21) \text {, y positivo/buena }(f=32) \text {. }\end{array}$ & $\begin{array}{l}\text { Ayuda y compañerismo }(f=263) \\
\text { Ayuda }(f=92) \text {, compañerismo }(f=59) \text {, compartir/intercambiar }(f=43) \text {, } \\
\text { comunicarte }(f=43) \text { aportaciones }(f=17) \text {, aconsejar }(f=6) \text {, colaborar }(f=3) \text {. }\end{array}$ \\
\hline $\begin{array}{l}\text { Ayuda y compañerismo }(f=278) \\
\text { Ayuda }(f=99) \text {, compañerismo }(f=74) \text {, colaborar }(f=34) \text {, compartir }(f=30) \text {, } \\
\text { aportaciones }(f=22) \text {, comunicar }(f=19) \text {. }\end{array}$ & $\begin{array}{l}\text { Utilidad para la mejora }(f=156) \\
\text { Utilidad }(f=39) \text {, mejorar }(f=20) \text {, oportunidad/posibilidad }(f=16) \text {, } \\
\text { importancia }(f=31) \text {, bueno/positivo }(f=50) .\end{array}$ \\
\hline $\begin{array}{l}\text { Reflexión }(f=249) \\
\text { Reflexionar }(f=78) \text {, discusión }(f=58) \text {, perspectiva }(f=37) \text {, dudas }(f=17) \text {, } \\
\text { puntos de vista }(f=8) \text {, opiniones }(f=6) \text {, evaluación }(f=41) \text {, conclusiones } \\
(f=4) \text {. }\end{array}$ & $\begin{array}{l}\text { Reflexión }(f=156) \\
\text { Reflexionar }(f=75) \text {, puntos de vista }(f=21) \text {, dudas }(f=22) \text {, opiniones } \\
(f=11) \text {, pensar }(f=11) \text {, analizar }(f=9) \text {, comparar }(f=4) \text {, crítico }(f=3) \text {. }\end{array}$ \\
\hline $\begin{array}{l}\text { Desarrollo docente }(f=122) \\
\text { Experiencia }(f=31) \text {, docente }(f=41) \text {, competencia }(f=18), \text { formación } \\
(f=17) \text {, ideas }(f=15) \text {. }\end{array}$ & $\begin{array}{l}\text { Desarrollo docente }(f=127) \\
\text { Experiencias }(f=67) \text {, aprendizaje }(f=32) \text {, ideas }(f=19) \text {, estrategias }(f=9) \text {. }\end{array}$ \\
\hline $\begin{array}{l}\text { Vivencia emocional }(f=33) \\
\text { Sentimientos }(f=23) \text {, vivencias }(f=10) \text {. }\end{array}$ & $\begin{array}{l}\text { Vivencia emocional }(f=28) \\
\text { Sentimientos }(f=17), \text { emociones }(f=5) \text {, pensamientos }(f=6) \text {. }\end{array}$ \\
\hline
\end{tabular}

Sentimientos $(f=23)$, vivencias $(f=10)$

Sentimientos $(f=17)$, emociones $(f=5)$, pensamientos $(f=6)$.

y el sentimiento de acompañamiento es otro de los aspectos que los participantes subrayan a la hora de identificar la utilidad del foro: "La considero una fuente de desahogo y comprensión" (A5).

En el Prácticum II se da un paso más destacando el pensamiento crítico como uno de los beneficios: "Tiene una utilidad muy positiva y práctica, ya que nos permite intercambiar experiencias, compartir ideas, conocer la realidad de otros centros y desarrollar nuestra capacidad crítica" (B10).

Entre todos los participantes, tan solo existe una persona de cada grupo (A17 del Prácticum I y B2 del Prácticum II) que opina que el foro no ha sido útil en su periodo de prácticas. El alumno del primer grupo no cree que haya necesitado el foro: “... no lo he necesitado, las dudas que he tenido o las cosas que me han pasado y me han extrañado durante las prácticas las he podido comentar con gente que también está haciendo prácticas, en persona" (A17).

Atendiendo a la estructura de los foros, se analizó la percepción del alumnado en cuanto a la pertinencia de cada uno de ellos:

- Primer foro: compartiendo experiencias y emociones.

- Segundo foro: prácticas de éxito para el futuro.

- Tercer foro: reflexionando para construir la profesionalidad del docente.

Aunque hay personas que expresan su preferencia por el tercer foro, el alumnado, en general, destaca el primer y el segundo foro como los más oportunos para enriquecer el periodo de prácticas: "Me han gustado más los dos primeros porque están más relacionados con lo que estaba haciendo en el momento" (A6). Estas percepciones expresadas por los estudiantes coinciden con el grado de participación real dado en cada foro.

"Los tres foros me parecen muy útiles, pero de destacar destacaría el segundo foro ya que es en el que más he participado. Además, creo que para el trabajo de PT es imprescindible tener conocimiento sobre diferentes materiales y estrategias, por lo que, me parece el foro más interesante respecto a estas prácticas" (B3).

En cuanto a las diferencias entre el Prácticum I y el Prácticum II, se puede observar cómo el segundo grupo, aunque muestra preferencia por el segundo foro, señala la complementariedad y la utilidad de todos los foros:
"La verdad que no me podría decantar por ninguno, ya que los tres han sido muy valiosos y útiles durante mis prácticas" (B11).

\section{El foro como espacio para la colaboración entre iguales}

Casi todos los participantes han destacado el foro como un espacio muy útil e importante para aprender con y de sus compañeros. En el primer grupo, se destaca el foro como herramienta de apoyo. Un participante argumenta: "Como estamos todos en la misma situación, creo que darnos ayuda mutua y resolver dudas entre nosotros es muy gratificante..." (A4).

También se describe como recurso para hacer frente a alguna de las limitaciones que tiene el periodo de prácticas. Una persona, por ejemplo, apunta el foro y la colaboración que se crea en este, como un recurso para superar los momentos de soledad que pueden aparecer en las prácticas: "Creo que es de gran importancia ya que es un momento en el que te puedes encontrar solo y sola, y necesitas tener apoyo a tu lado" (A8).

En el Prácticum II, no le dan solamente importancia al apoyo, sino también a "... fomentar la comunicación y cohesión de grupo...” (B10), a “... estar en contacto con personas que están en la misma situación” (B12), a “... conversar con personas del mismo gremio" (B9). Es decir, a crear y mantener la relación entre iguales: "El foro ha sido una herramienta con la que hemos tenido relación durante el periodo de prácticas, si no se hubiese utilizado cada experiencia se hubiese quedado para uno mismo" (B1) y "La verdad que hasta ahora nunca me lo había planteado, pero es una manera muy óptima de abrir el círculo..." (B11).

Mientras en el Prácticum II todas las respuestas han sido positivas, dos participantes del Prácticum I no han valorado el foro como una herramienta importante para la colaboración señalando lo siguiente: "He tenido la suerte de hacer las prácticas con compañeros y por ello no le he dado tanta importancia al foro, ya que podía compartir mis dudas con ellos" (A13) y "No mucho, ya que por mi parte ya lo podía comentar por otros medios con la misma gente" (A17). 


\section{El foro para la regulación emocional y el aprendizaje basado en la experiencia}

El alumnado de los Prácticum I y II muestra un amplio consenso en cuanto a la utilidad del foro como herramienta para la regulación emocional y el aprendizaje basado en la experiencia. El $86.20 \%$ de los estudiantes coincide en que la participación activa que han creado en los foros se ha llevado a cabo en un ambiente de aprendizaje relajado y de apoyo, ayudándose los unos a los otros y aprendiendo desde diferentes puntos de vista: "Me ha parecido una oportunidad muy buena que a lo mejor si no la hubiésemos tenido no hubiese sido igual. Es una buena manera de desahogarte, poder ayudar a resolver cuestiones y aprender de los demás" (A4).

Además, son numerosas las ocasiones en las que el alumnado destaca la ayuda recibida en este espacio colaborativo: "Siento que tenía a alguien que me escuchaba y me podía aconsejar y, en algunos momentos, era algo que necesitaba" (A8).

Ambos grupos comparten la misma opinión y además inciden en la participación activa: "Personalmente, me ha servido mucho el foro, ya que, en las situaciones difíciles, he podido compartir mis sentimientos, leer las respuestas de mis compañeros/as y sentirme mejor, desahogarme" (B12), "Lo valoro positivamente, ya que hemos tomado parte activamente en él y eso significa que los puntos a debatir han sido de gran interés (B1)".

Sin embargo, dos participantes de cada grupo -4 en total - hacen referencia a la dificultad de publicar y compartir sentimientos: "Un poco complicado porque al final todo se ve y los compañeros leen lo que estás escribiendo y quizá no es la mejor manera de sacar tus sentimientos cuando hay tantas personas que pueden leerlos" (A6) y "Creo que compartir estados emocionales a través de los foros es más difícil, por lo tanto, pienso que la parte de compartir emociones debe ser presencial" (B7).

Esta opinión contrasta con la del participante que cree que el foro puede resultar útil a ciertas personas: "Yo no tengo ningún tipo de problema para expresar mis pensamientos y sentimientos. Sin embargo, quizás otra persona pueda tener dificultades y el foro puede ser un buen modo para soltarse un poco" (A11).

\section{El foro como espacio para la reflexión}

Existe un amplio consenso (96.55\%) entre el alumnado a la hora de indicar que el uso de los foros ha sido de gran ayuda para reflexionar sobre su práctica docente. Además, el 89.65\% reconoce la importancia de la reflexión para la mejora docente.

Subrayan la utilidad del foro en este sentido, ya que contar con diferentes perspectivas y analizar las situaciones en profundidad les ha ayudado a mejorar su práctica docente durante el Prácticum: "Lo que más ayuda en la práctica es la actitud activa ante una situación, pero ver los diferentes puntos de vista de mis compañeros también me ha ayudado a mejorar a la hora de tomar parte en la clase" (A2), "Sí, me ha ayudado. Analizando la situación en profundidad y buscando así una solución en consonancia al problema" (B4).

Además, en ambos grupos definen la reflexión como una herramienta para la mejora continua de uno mismo, ya sea revisando las acciones propias como recibiendo feedback de otro compañero: "Sí. Me ha gustado cuestionarme en todo momento, poner en duda mis actos y mejorar como docente" (A5), “... sobre todo el hecho de recibir feedback por parte de mis compañeros y conocer sus experiencias" (B10).

Son numerosas las ocasiones en las que los estudiantes apuntan que el foro les ayuda a conocer y contrastar diferentes puntos de vista y opiniones, distinguiendo las percepciones de uno mismo de las del resto de sus compañeros: “... haciendo esa comparación con mis compañeros de la que sacabas bastantes puntos a mejorar de tu actuación..." (A14), "Me he puesto en las situaciones de mis compañeros, me ha proporcionado otros puntos de vista y aprendizajes" (B7). Asimismo, evidencian que el foro les ha ayudado a profundizar en el grado de reflexión mediante diferentes estrategias: "Creo que me ha ayudado bastante porque a medida que iba escribiendo en el foro me daba cuenta de ciertas cosas, que quizás en el momento no" (A3), "Me ha hecho hacerme preguntas que quizás por mí mismo no me haría" (B8).

En definitiva, el alumnado destaca que el foro es una herramienta muy útil para favorecer la reflexión y considerar la importancia de esta. Además, describen la herramienta como una oportunidad para observar la realidad educativa desde una visión más amplia y mediante el pensamiento crítico: "Me ha ayudado bastante, ya que creo, que en la docencia es muy importante reflexionar. Y con este foro me he dado cuenta aún más" (A3), “... me ha dado una visión más amplia y crítica sobre la escuela y el profesor docente y creo que he podido aprender desde mis compañeros nuevas lecciones que me serán muy útiles" (B11).

Se perciben opiniones especialmente diferentes en el grupo de estudiantes del Prácticum II con una mayor formación y práctica profesional. Existe un número significativo de estudiantes (58.3\%) en este grupo que afirma que en estas prácticas realizadas su aprendizaje ha sido mayor gracias al foro. Esto queda reflejado en las siguientes afirmaciones: “... en las anteriores prácticas, no tuve la oportunidad de intercambiar mis experiencias con mis compañeros/as de clase. Tampoco pude ver qué otros proyectos y metodologías se llevan a cabo en otras escuelas. Por lo tanto, en la mención de PT, he podido analizar otros puntos de vista sobre las escuelas y la educación" (B12).

En algún caso, también aluden a la naturaleza de la propia mención como razón para profundizar en su reflexión docente: "Este trabajo como PT te hace reflexionar mucho sobre la vida" (B5). 


\section{Nivel de satisfacción en el uso del foro}

El 93.10\% de los participantes afirman que recomendarían el foro a otros estudiantes en prácticas y reconocen que es una herramienta valiosa para el prácticum: "Sí, creo que es un método que mejora la experiencia de las prácticas" (A8).

Ambos grupos coinciden $\mathrm{y}$, entre las diferentes respuestas afirmativas, se expone que el foro es una herramienta muy útil y lo valoran como una oportunidad para ampliar sus conocimientos, reflexionar, expresar opiniones y sentimientos; y, por último, ser agentes de apoyo, así como sentirse apoyados por sus compañeros.

"La utilidad del foro ha sido muy positiva. Anteriormente, no tuve la oportunidad de compartir mis experiencias y sentimientos sobre las prácticas con mis compañeros/as. Gracias a este foro, he podido expresar lo que he sentido en las prácticas (tanto lo positivo, como lo negativo), leer las diferentes metodologías que se utilizan en otros colegios, pensar sobre las adecuadas formas de educar, etc. Por todo ello, recomendaría el uso de los foros en las prácticas" (B12).

Además, tal y como expresa un alumno, su recomendación va aún más allá: “... buenísima idea. Creo que esto se debería de hacer con todos los profesionales ya trabajadores, y no solo en la universidad" (B6).

\section{Discusión}

El estudio realizado ha permitido constatar el interés del alumnado por emplear recursos tecnológicos, como los foros online, y su implicación en el uso durante la realización de las prácticas como docentes de Educación Primaria. Asimismo, se observa un amplio consenso entre los estudiantes participantes en considerar el foro una herramienta útil al servicio del aprendizaje. La experiencia permite corroborar, de este modo, que las plataformas de educación ofrecen amplias posibilidades al servicio del aprendizaje y de la personalización de la respuesta educativa a las necesidades de los estudiantes (Gros \& Cano, 2018).

Los participantes en el estudio coinciden en identificar entre las principales utilidades del foro, su potencial para favorecer la colaboración y el aprendizaje entre iguales, la reflexión sobre la acción para mejorar la práctica profesional, así como la gestión y regulación emocional ante los sucesos que se desencadenan diariamente en los centros escolares. Este hecho sugiere que los propios estudiantes son conscientes de las ventajas que supone el uso de esta herramienta.

De este modo, se observa que el foro online aplicado al prácticum permite lograr dos metas de relevancia en la formación de los futuros docentes, recogidas por Coll et al. (2018). Por un lado, permite incorporar las TIC para promover, interconectar e individualizar el aprendizaje, y por otro, favorece un espacio para reflexionar colaborativamente con otros compañeros sobre las situaciones que la práctica arroja y así poder gestionar conjuntamente y de la manera más eficaz la respuesta más conveniente a cada una de ellas.

Además, la aplicación de este recurso resuelve una de las mayores dificultades que entrañan los periodos de prácticas: dar soporte y apoyo continuo sobre las realidades vividas diariamente en el centro. El foro, que permite interaccionar con otros compañeros y los tutores de la universidad, posibilita compartir inquietudes y reflexiones profesionales, consolidando el desarrollo de sus competencias profesionales. Asimismo, se agiliza el diseño y la implementación de intervenciones educativas positivas por parte de los estudiantes en prácticas, enriqueciendo su experiencia y cooperando con los centros educativos que los acogen. En definitiva, resulta una buena solución para garantizar buenas experiencias de aprendizaje entre los futuros docentes y permite tender puentes y conexiones directas entre la universidad y la escuela; cuestión indispensable en los periodos de prácticas (Mauri, Clarà, Colomina \& Onrubia, 2016).

La experiencia ha contribuido, además, a constatar otras importantes ventajas. Por un lado, de acuerdo con las aportaciones de Gros y Cano (2018), la plataforma Moodle permite registrar el tipo de interacciones que hace el estudiante en ella, facilitando así al profesorado universitario avanzar en el modo en que se pueden mejorar los aprendizajes de los estudiantes. Asimismo, la interacción rápida y ágil que ofrece ayuda a tomar medidas en el momento oportuno, para adaptarse a las necesidades específicas de cada estudiante. Es decir, las analíticas de aprendizaje que es posible realizar gracias al registro que hace Moodle, pueden desembocar en la construcción de aprendizajes personalizados y adaptados a las necesidades que la práctica docente genera a los estudiantes.

El análisis de las valoraciones realizadas por los estudiantes que tomaron parte en la experiencia, permite extraer algunas conclusiones de interés para seguir optimizando el uso de este recurso:

- Favorece la colaboración, el apoyo mutuo y el aprendizaje entre iguales, de acuerdo con las evidencias obtenidas por Romero et al. (2013). En este sentido los estudiantes valoran muy positivamente la interacción con sus compañeros y su impacto sobre el aprovechamiento de su estancia en los centros escolares. Los estudiantes que no han enfatizado esta utilidad, una minoría entre el total de participantes, aseguran, sin embargo, no haberla necesitado dado que ya disponían del apoyo de compañeros en el propio centro. Por tanto, su escasa valoración parece no deberse a la no pertinencia de esta utilidad, sino a la oportunidad particular y casuística de haber podido realizar prácticas en compañía de otros estudiantes.

- El clima relajado e informal encontrado en los foros, y el ambiente de apoyo al que se refieren los estudiantes al ser consultados, facilita la construcción de aprendizajes significativos que consoliden el desarrollo de las competencias del profesorado. 
- Una alta participación en los foros focalizados en el análisis de las experiencias educativas de éxito permite entrever entre el alumnado un interés y compromiso con la mejora de la calidad educativa, con la innovación y con la necesidad de cambio para responder a las necesidades educativas vigentes, cuestión vital que hay que desarrollar en la formación inicial para consolidar prácticas profesionales éticas y coherentes con los desafíos educativos.

- Las posibilidades contrastadas para favorecer la reflexión habrán de ser consideradas para futuras experiencias de prácticas. La reflexión sobre la acción se consolida como un itinerario seguro y fiable hacia el desarrollo docente y mejora de la práctica profesional (Schön, 1983; Altet, 2005; Charlier, 2005; Marchesi \& Martín, 2014; Martínez-Izaguirre et al., 2018; Martínez-Izaguirre, Diaz-Iso \& Barrenetxea, 2018).

- La alta participación en el foro orientado a compartir sentimientos y pensamientos sobre las prácticas, respalda la necesidad de apoyo y acompañamiento durante el aprendizaje, incluido el que se da en los procesos de prácticas, que se ve limitado asiduamente por la distancia espaciotemporal con compañeros y tutores de universidad. Asimismo, consolida la necesidad de promover la educación emocional de los futuros docentes y el desarrollo de un nivel de competencia básico en gestión emocional (Martínez-Izaguirre et al., 2018).

- Aunque el foro promueve un espacio inigualable para desarrollo e implementación de la competencia para la gestión emocional, y los estudiantes se han mostrado muy motivados por participar en el foro destinado a tal fin, se observa en algunas respuestas recogidas, la tarea pendiente existente a día de hoy en alfabetizar en este ámbito a los estudiantes. Así pues, existen algunos alumnos que no se sienten cómodos compartiendo experiencias, sentimientos y emociones sobre realidades vividas.

Tal y como plantean Fernández-Berrocal \& Extremera (2009), las habilidades emocionales se relacionan con la felicidad, el funcionamiento social y el bienestar. Por tanto, parece resultar esencial poner atención al desarrollo emocional en la formación humana, más aún en la formación de futuros docentes, donde la competencia emocional es indispensable para cumplir con las funciones que entraña la profesión. Mientras en las escuelas del siglo xx el éxito docente se asociaba al rendimiento escolar de su alumnado, en las escuelas del siglo xxi esto ya no es suficiente. El éxito docente se alcanza al formar ciudadanos capaces de integrarse en la sociedad, que dispongan de herramientas sociales y emocionales que les permitan responder ante las demandas de la vida cotidiana (Cabello, Ruiz-Aranda \& Fernández-Berrocal, 2009)

Además, la seguridad que ofrece la gestión eficaz de las emociones en los docentes incrementa su capacidad para ayudar, empatizar y aceptar a los demás (Camacho \& Mendías, 2005); permite responder de una manera más eficaz a la creciente violencia escolar (Fernández Herrería \& López López, 2007); y ayuda en el aprendizaje y la salud mental y física de su alumnado, así como sobre su rendimiento académico (Belando, 1999).

Los participantes coinciden en que el foro les brinda una oportunidad para estructurar, focalizar y compartir experiencias que facilitan el manejo de diferentes puntos de vista y la localización de respuestas a las dudas surgidas durante el periodo de prácticas, ayudándoles así en su práctica docente.

A la luz de los beneficios encontrados en la aplicación de esta herramienta, aunque la experiencia se ha desarrollado en el Grado de Educación Primaria, podría resultar útil aprovechar las posibilidades que ofrece el foro en las prácticas en otros contextos o ramas profesionales.

\section{Referencias}

Altet, M. (2005). La competencia del maestro profesional o la importancia de saber analizar las prácticas. En L. Paquay, M. Altet, E. Charlier y P. Perrenoud (coords.), La formación profesional del maestro. Estrategias y competencias (pp. 33-48). México: Fondo de Cultura Económica.

Belando, M. R. (1999). Los profesores del siglo xxi y la calidad de la enseñanza universitaria: en torno a la formación. Revista Electrónica Interuniversitaria de Formación del Profesorado, 2(1), 99-106.

Cabello, R., Ruiz-Aranda, D. \& Fernández-Berrocal, P. (2009). Docentes emocionalmente inteligentes. Revista Electrónica Interuniversitaria de Formación del Profesorado, 13(1), 41-49.

Camacho, H. \& Mendías, A. M. (2005). Aportación a la mejora de la calidad en la formación de maestros. Revista Iberoamericana de Educación, 35(6), 2-18.

Carbonell, J. (2005). El profesorado y la innovación educativa. En P. Cañal de León (coord.), La innovación educativa (pp. 11-27). Madrid: Akal.

Charlier, E. (2005). Former des enseignants-professionnels pour une formation continuée articulée à la pratique. En L. Paquay, M. Altet, E. Charlier, y F. Perrenoud (eds.), Former des enseignants professionnels. Quelles stratégies? Quelles compétences? (pp. 97-117). Namur: De Boeck Supérieur.

Coll, C., Mauri, T., Colomina, R. M., Engel, A., Oller, J., Onrubia, J. \& Rochera, M. J. (2018). Hacia una educación distribuida e interconectada. Algunas implicaciones para la formación de los docentes. En T. Lleixà,, B. Gros, T. Mauri, y J. L. Medina (coords.). Educación 2018-2020. Retos, tendencias y compromisos (pp. 27-32). Barcelona: IRE-UB.

Durán, D. \& Vidal, V. (2004). Tutoría entre iguales: De la teoría a la práctica: Un método de aprendizaje cooperativo para la diversidad en secundaria. Barcelona: Graó.

Escobar-Pérez, J. \& Cuervo-Martínez, A. (2008). Validez de contenido y juicio de expertos: una aproximación a su utilización. Avances en Medición, 6, 27-36.

Escudero, J. M. (2006). La formación del profesorado y la garantía del derecho a una buena educación para 
todos. En J. M. Escudero, y A. L. Gómez (eds.), La formación del profesorado y la mejora de la educación (pp. 21-51). Barcelona: Octaedro.

Fernández-Berrocal, P. \& Extremera, N. (2009). La inteligencia emocional y el estudio de la felicidad. Revista Interuniversitaria de Formación del Profesorado, 66, 85-108.

Fernández Herrería, A. \& López López, M. C. (2007). La inclusión del componente emocional en la formación inicial de maestros. Una experiencia para el desarrollo de la conciencia sensorial. Revista Iberoamericana de Educación, 43(3), 1-12.

Gisbert, M. (2013). Nuevos escenarios para los aprendices digitales en la universidad. Aloma: Revista de Psicologia, Ciències de l'educació i de l'esport, 31, 55-64.

Gros, B. \& Cano, E. (2018). El uso de las analíticas de aprendizaje para la mejora de la formación. En T. Lleixà, B. Gros, T. Mauri, y J. L. Medina (coords.). Educación 2018-2020. Retos, tendencias y compromisos (pp. 45-50). Barcelona: IRE-UB.

Kokotsaki, D., \& Hallam, S. (2007). Higher education music students' perceptions of the benefits of participative music making. Music Education Research, 9, 93-109. doi: 10.1080/14613800601127577

Loncar, M., Barrett, N. E., \& Liu, G. Z. (2014). Towards the refinement of forum and asynchronous online discussion in educational contexts worldwide: Trends and investigative approaches within a dominant research paradigm. Computers \& Education, 73, 93110E. doi: 10.1016/j.compedu.2013.12.007

Marchesi, A., \& Martín, E. (2014). Calidad de la enseñanza en tiempos de crisis. Madrid: Alianza Editorial.

Martínez-Izaguirre, M., Diaz-Iso, A. \& BarrenetxeaMinguez, L. (abril, 2018). El uso de los foros en el Practicum para el desarrollo profesional de los estudiantes del Grado de Educación Primaria. Trabajo presentado en VIII Jornada Universitaria de Innovación y Calidad: Los espacios (físicos y virtuales) al servicio del aprendizaje. Recuperado de https://bit. ly/2TCkCPk

Martínez-Izaguirre, M., Yániz-Álvarez de Eulate, C. \& Villardón-Gallego, L. (2018). Autoevaluación y reflexión docente para la mejora de la competencia profesional del profesorado en la sociedad del cono- cimiento. RED. Revista de Educación a Distancia. doi: 10.6018/red/56/10

Mauri, T., Clarà, C., Colomina, R. \& Onrubia, J. (2016). Educational assistance to improve reflective practice among student teachers. Electronic Journal of Research in Educational Psychology, 14, 287-309. doi:10.14204/ ejrep.39.15070

McFarlane, R., Spes-Skrbis, M. \& Taib, A. (2017). Let's Chat - A fresh take on the invaluable role of peer-topeer conversation in student engagement, participation and inclusion. Student Success, 8(2), 107-111. doi: $10.5204 /$ ssj.v8i2.388

Ornelas, D. (2007). El uso del foro de discusión virtual en la enseñanza. Revista Iberoamericana de Educación, 44, 4-10.

Parks-Stamm, E. J., Zafonte, M. \& Palenque, S. M. (2017). The effects of instructor participation and class size on student participation in an online class discussion forum. British Journal of Educational Technology, 48(6), 1250-1259.

Romero, C., López, M. I., Luna, J. M. \& Ventura, S. (2013). Predicting students' final performance from participation in on-line discussion forums. Computers \& Education, 68, 458-472. doi: 10.1016/j.compedu.2013.06.009

Sanabria, A., \& Hernández, C. M. (2011). Percepción de los estudiantes y profesores sobre el uso de las TIC en los procesos de cambio e innovación en la enseñanza superior. Aloma: Revista de Psicologia, Ciències de l'educació i de l'esport Blanquerna, 29, 273-290.

Schön, D. A. (1983). The reflective practitioner: How professionals think in action. New York: Basic Books.

Tan, K. E. (2017). Using online discussion forums to support learning of paraphrasing. British Journal of Educational Technology, 48(6), 1239-1249. doi: 10.1111/bjet. 12491

Tirado, R., Hernando, Á., \& Aguaded, J. I. (2015). The effect of centralization and cohesion on the social construction of knowledge in discussion forums. Interactive Learning Environments, 23(3), 293-316. doi: 10.1080/10494820.2012.745437

Topping, K. J. (1996). The effectiveness of peer tutoring in further and higher education: A typology and review of the literature. Higher education, 32(3), 321-345. 